\title{
COVID-19 Digestive Symptoms Mimicking Internal Hernia Presentation After Roux-en-Y-Gastric Bypass; Comment on "Internal Hernia in the Times of COVID-19: to Laparoscope or Not to Laparoscope?"
}

\author{
Louis Betton $^{1}$ - Deborah Benchetrit ${ }^{2}$ Judith Aron-Wisnewsky ${ }^{3,4,5}$ - Jean-Michel Oppert ${ }^{3,4,5}$ - Adriana Torcivia ${ }^{1}$. \\ Jean-Christophe Vaillant ${ }^{1,3} \cdot$ Laurent Genser $^{1,3,5}$ (D) \\ Published online: 8 May 2020 \\ (C) Springer Science+Business Media, LLC, part of Springer Nature 2020
}

Keywords Bypass · Obesity · Bariatric · Bypass · Coronavirus disease 2019 (COVID-19) · SARS-CoV-2 · Internal hernia · CT

We read with interest the manuscript by Dr. Singhal and share similar opinion regarding the difficulties to balance the benefits and risks of a surgical exploration in case of suspected internal hernia (IH) after Roux-en-Y gastric bypass (RYGB) during the present context of COVID-19 pandemic [1].

Indeed, as stated by Dr. Singhal, internal hernia (IH) represents a frequent cause of obstruction after RYGB leading to potentially severe consequences [2]. IH clinical presentation is nonspecific and commonly associates intermittent gastrointestinal symptoms usually worsened by food intake, subsequently leading to anorexia [2]. Noteworthy, COVID-19 infection can also be diagnosed upon various nonspecific digestive symptoms in 3 to $79 \%$ of cases according to the published series. These symptoms include anorexia (39.9-50.2\%), vomiting (3.6-66.7\%), diarrhea (2-49.5\%), and/or abdominal pain $(2.2-6 \%)$ [3]. However, little is currently known on these

Laurent Genser

laurent.genser@aphp.fr

Louis Betton

betton.louis@gmail.com

Deborah Benchetrit

deborah.benchetrit@aphp.fr

Judith Aron-Wisnewsky

judith.aron-wisnewsky@aphp.fr

Jean-Michel Oppert

jean-michel.oppert@aphp.fr

Adriana Torcivia

adriana.torcivia@aphp.fr

Jean-Christophe Vaillant

jean-christophe.vaillant@aphp.fr gastrointestinal symptoms in the context of patients previously operated from bariatric surgery and especially after procedures that modify the gastrointestinal tract anatomy (i.e., RYGB).

We recently admitted a 57-year-old female patient who had undergone a RYGB 5 years and 1 month earlier for grade III obesity (112 kg; body mass index: $42 \mathrm{~kg} / \mathrm{m}^{2}$ ) associated with type 2 diabetes, hypertension, and obstructive sleep apnea syndrome. Based on the patient charts, mesenteric defects were left open during the procedure. The initial postoperative course and the follow-up were uneventful. At her planned 5year postoperative follow-up appointment (February 2020), she weighed $85 \mathrm{~kg}$ (BMI: $31.8 \mathrm{~kg} / \mathrm{m}^{2}$ ) and had a normal physical examination. Two months later (April 2020), she presented with transient and unspecific episodes of acute abdominal pain aggravated by food intake followed by anorexia and

1 Assistance Publique-Hôpitaux de Paris (AP-HP), Department of Hepato-Biliary and Pancreatic Surgery, Pitié-Salpêtrière University Hospital, Sorbonne Université, 47-83 boulevard de l'Hôpital, 75013 Paris, France

2 Assistance Publique-Hôpitaux de Paris (AP-HP), Department of anaesthesia and intensive care,Pitié-Salpêtrière University Hospital, Sorbonne Université, 47-83 boulevard de l'Hôpital, 75013 Paris, France

3 Institute of Cardiometabolism and Nutrition (ICAN), Sorbonne Université, 47-83 boulevard de l'Hôpital, 75013 Paris, France

4 Assistance Publique-Hôpitaux de Paris (AP-HP), Nutrition Department, Pitié-Salpêtrière University Hospital, Sorbonne Université, 47-83 boulevard de l'Hôpital, 75013 Paris, France

5 NutriOmics team, INSERM UMRS U1269, Sorbonne Universités, Paris, France 

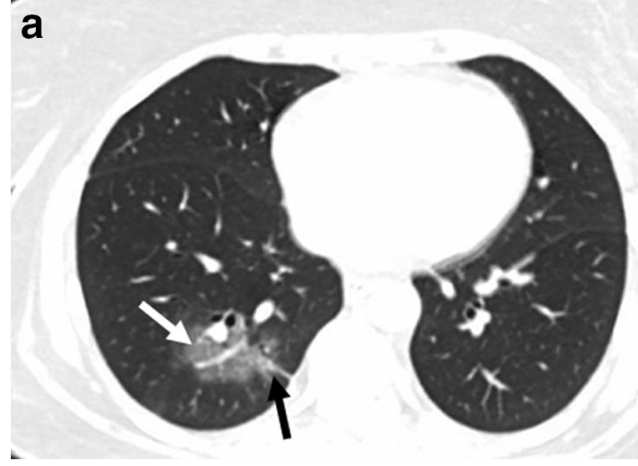

Fig. 1 a Axial chest CT showing features of COVID-19: multiple ground-glass opacities with interlobular septal thickening (white arrow) and vascular enlargement (black arrow) in the peripheral of the right lung.

absence of flatus. She displayed neither signs of pulmonary infection nor general signs of severity or fever. On laboratory tests, white blood cell count and PCT were within normal ranges, and CRP was slightly increased $(13.7 \mathrm{mg} / \mathrm{L} N<$ $5 \mathrm{mg} / \mathrm{l})$. Since the clinical history was highly suggestive of an $\mathrm{IH}$, we performed an emergent abdominal CT scan. While abdomen imaging was normal, we incidentally found, on chest CT images, signs typically suggestive of COVID-19 infection (Fig. 1) [4]. The nasopharyngeal swab, analyzed by real-time polymerase chain reaction (RT-PCR) testing, proved positive for severe acute respiratory syndromecoronavirus 2 (SARS-CoV-2; i.e., COVID 19). The patient was hospitalized and benefitted from COVID-19 symptomatic treatment and further discharged after 2 days of observation with favorable clinical evolution. Two weeks later, she no longer complained from any digestive symptom and was recovering in spite of persisting asthenia.

RYGB is a procedure that allows an important and sustainable weight loss [5]. It enables major improvement in obesity and its related complications, thus leading to reduced premature death [6]. Nevertheless, since the mean BMI loss 12 years after RYGB reaches $11.5 \mathrm{~kg} / \mathrm{m}^{2}$, a non-negligible amount of patients remain with a postoperative BMI $\geq 30 \mathrm{~kg} / \mathrm{m}^{2}$. Importantly, obesity whatever its grade represents a known risk factor of severe forms of COVID-19, whether or not it is associated with other demonstrated risks factors such as hypertension or type 2 diabetes [7].

Findings from this case report combined with recent knowledge on the various clinical presentations of COVID19 may justify a systematic chest and abdominal CT in patients operated from RYGB, seen in the emergency room with acute and nonspecific digestive symptoms. This would help provide optimal management and avoid potentially dangerous surgical procedure in the times of this pandemic.

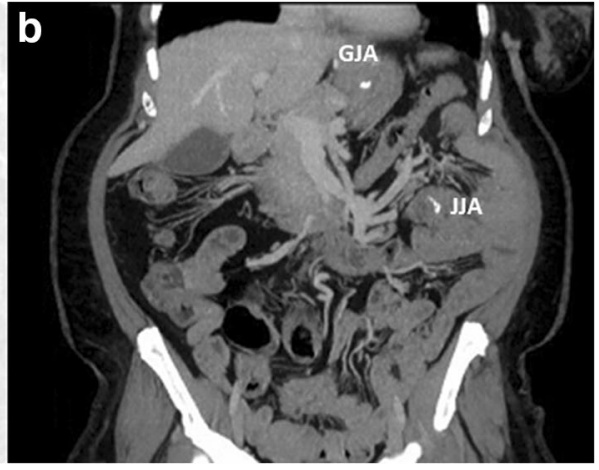

b Coronal abdominal CT scanner showing no direct/indirect signs of internal hernia or obstructions. GJA, gastro-jejunal anastomosis; JJA, jejuno-jejunal anastomosis

\section{Compliance with Ethical Standards}

Conflict of Interest The authors declare that they have no conflict of interest.

Ethical Approval For this type of study, formal consent is not required.

Informed Consent Informed consent was obtained from all individual participants included in the study.

\section{References}

1. Singhal R. Internal Hernia in the Times of COVID-19: to Laparoscope or Not to Laparoscope? OBES SURG [Internet]. 2020 [cited 2020 Apr 17]; Available from: https://doi.org/10.1007/ s11695-020-04598-x

2. Stenberg E, Szabo E, Ågren G, et al. Closure of mesenteric defects in laparoscopic gastric bypass: a multicentre, randomised, parallel, open-label trial. Lancet. 2016;387:1397-404.

3. Tian Y, Rong L, Nian W, et al. Review article: gastrointestinal features in COVID-19 and the possibility of faecal transmission. Aliment Pharmacol Ther. 2020;51:843-51.

4. Li M. Chest CT features and their role in COVID-19. Radiol Infect Dis 2020;

5. Adams TD, Davidson LE, Litwin SE, et al. Weight and metabolic outcomes 12 years after gastric bypass. N Engl J Med. 2017;377: $1143-55$.

6. Global BMI Mortality Collaboration. Body-mass index and all-cause mortality: individual-participant-data meta-analysis of 239 prospective studies in four continents. Lancet. 2016;388(10046):776-86. https://doi.org/10.1016/S0140-6736(16)30175-1.

7. Simonnet A, Chetboun M, Poissy J, Raverdy V, Noulette J, Duhamel A, et al. High prevalence of obesity in severe acute respiratory syndrome coronavirus-2 (SARS-CoV-2) requiring invasive mechanical ventilation. Obesity (Silver Spring). 2020;

Publisher's Note Springer Nature remains neutral with regard to jurisdictional claims in published maps and institutional affiliations. 